\title{
Corrigendum to "Detecting Key Genes Regulated by miRNAs in Dysfunctional Crosstalk Pathway of Myasthenia Gravis"
}

\author{
Yuze Cao, ${ }^{1,2}$ Jianjian Wang, ${ }^{1}$ Huixue Zhang, ${ }^{1}$ Qinghua Tian, ${ }^{1}$ Lixia Chen, ${ }^{1}$ \\ Shangwei Ning, ${ }^{3}$ Peifang Liu, ${ }^{1}$ Xuesong Sun, ${ }^{1}$ Xiaoyu Lu, ${ }^{1}$ Chang Song, ${ }^{1}$ Shuai Zhang, \\ Bo Xiao, ${ }^{2}$ and Lihua Wang ${ }^{1}$ \\ ${ }^{1}$ Department of Neurology, The Second Affiliated Hospital, Harbin Medical University, Harbin, Heilongjiang 150081, China \\ ${ }^{2}$ Department of Neurology, Xiangya Hospital, Central South University, Changsha, Hunan 410008, China \\ ${ }^{3}$ College of Bioinformatics Science and Technology, Harbin Medical University, Harbin, Heilongjiang 150081, China
}

Correspondence should be addressed to Bo Xiao; xiaobo_xy@126.com and Lihua Wang; wanglh211@163.com

Received 4 January 2017; Accepted 19 January 2017; Published 14 August 2017

Copyright (C) 2017 Yuze Cao et al. This is an open access article distributed under the Creative Commons Attribution License, which permits unrestricted use, distribution, and reproduction in any medium, provided the original work is properly cited.

In the article titled "Detecting Key Genes Regulated by miRNAs in Dysfunctional Crosstalk Pathway of Myasthenia Gravis" [1], it was not clearly stated how to identify dysfunctional pathways in myasthenia gravis (MG), as raised by Dr. Panse in [2]. The authors clarified how to overcome the issue in their response [3]. Accordingly, the Results and Discussion sections are corrected as follows.

In the Results section, "Since miRNAs are negative regulators of mRNAs, pathways enriched for differentially expressed mRNAs were used to filter predicted targets based on inverse miRNA-mRNA regulation. The intersections of $\mathrm{P}_{1}$ $+\mathrm{P}_{4}$ and $\mathrm{P}_{2}+\mathrm{P}_{3}$ were defined as up- and downregulated pathways, respectively" should be corrected to "Since miRNAs are negative regulators of mRNAs, pathways enriched for differentially expressed mRNAs were used to filter predicted targets based on inverse miRNA-mRNA regulation. In brief, we applied pathway filter analyses instead of using miRNAmRNA regulatory pairs directly. The intersections of $\mathrm{P}_{1}+\mathrm{P}_{4}$ and $\mathrm{P}_{2}+\mathrm{P}_{3}$ were defined as up- and downregulated pathways, respectively."

In the Discussion section, "However, some MG patients are unresponsive to conventional therapies or suffer adverse reactions from long-term use of immunomodulatory drugs such as steroids or immunosuppressants" should be corrected to "However, some MG patients are unresponsive to conventional therapies or suffer adverse reactions from long-term use of immunomodulatory drugs such as steroids or immunosuppressants. The category of MG patients is complex, which could be classified according to the antibody specificity, age at onset, type of course, and thymus histology. Therefore, we do not consider anti-AChR-positive and antiAChR-negative patients specifically."

\section{References}

[1] Y. Cao, J. Wang, H. Zhang et al., "Detecting key genes regulated by miRNAs in dysfunctional crosstalk pathway of myasthenia gravis," BioMed Research International, vol. 2015, Article ID 724715, 10 pages, 2015.

[2] R. Le Panse, "Comment on 'detecting key genes regulated by miRNAs in dysfunctional crosstalk pathway of myasthenia gravis,' BioMed Research International, vol. 2017, Article ID 6950308, 1 page, 2017.

[3] Y. Cao, J. Wang, H. Zhang et al., "Response to: comment on 'detecting key genes regulated by miRNAs in dysfunctional crosstalk pathway of myasthenia gravis," BioMed Research International, vol. 2017, Article ID 5359434, 2 pages, 2017. 

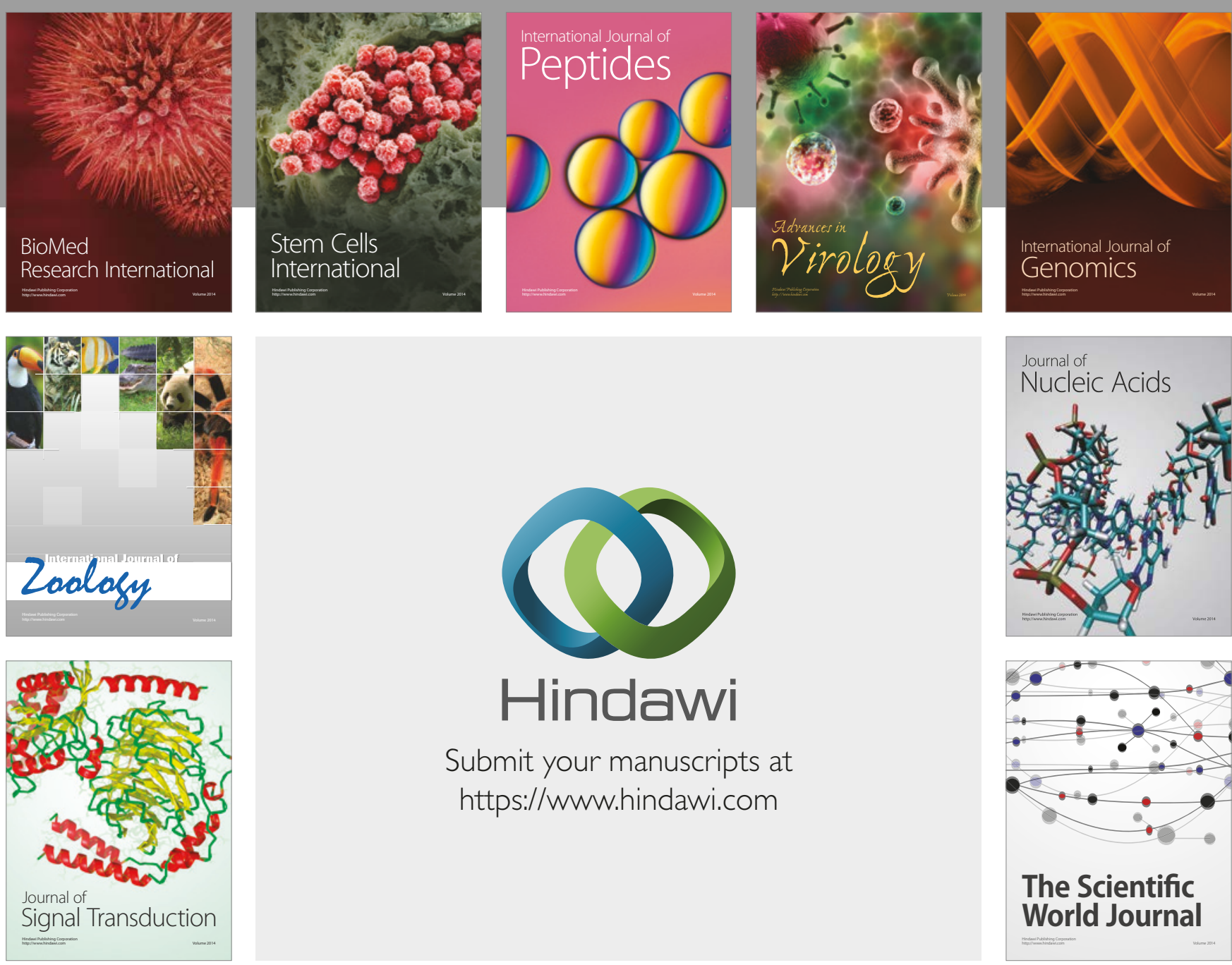

Submit your manuscripts at

https://www.hindawi.com
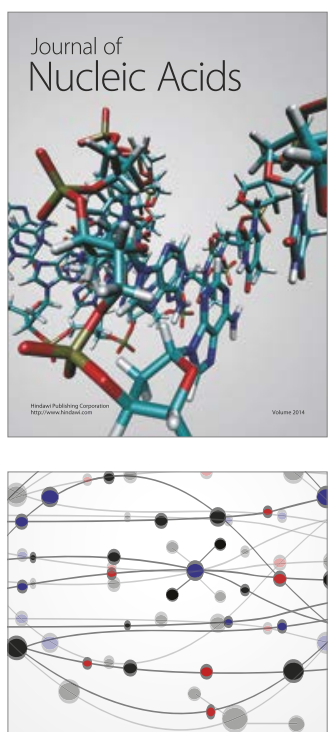

The Scientific World Journal

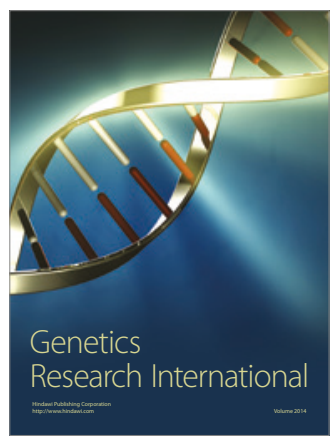

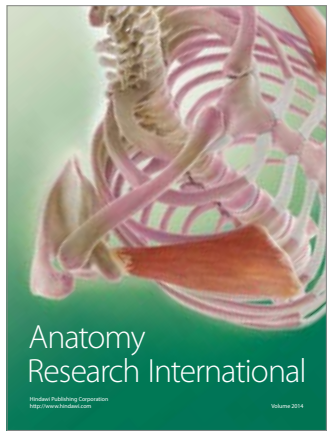

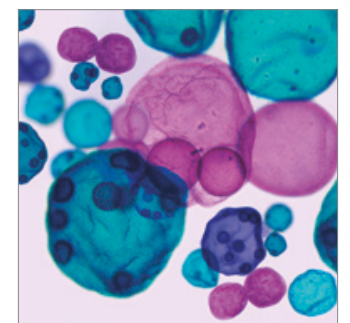

International Journal of Microbiology
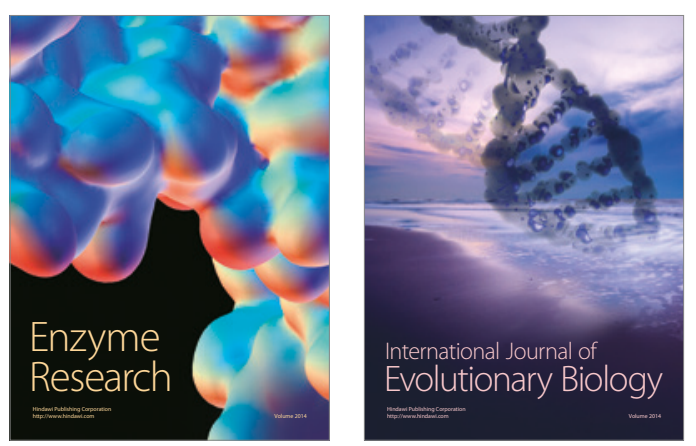
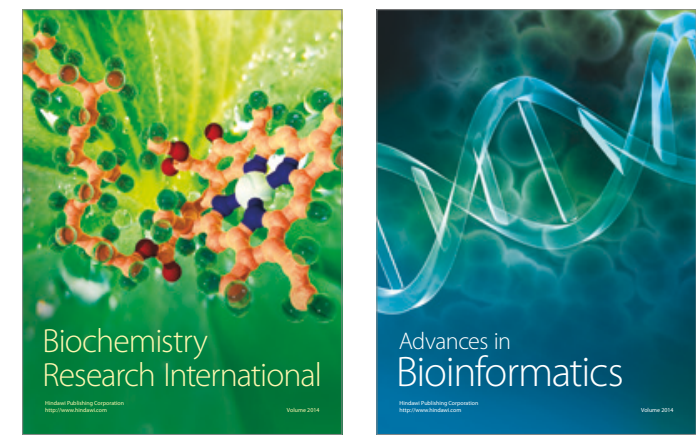

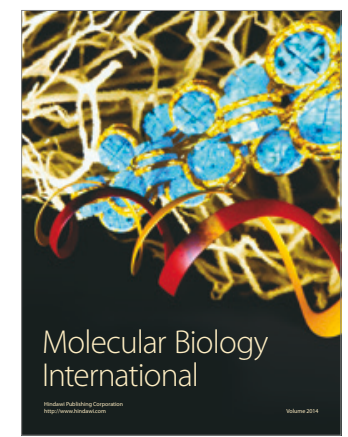

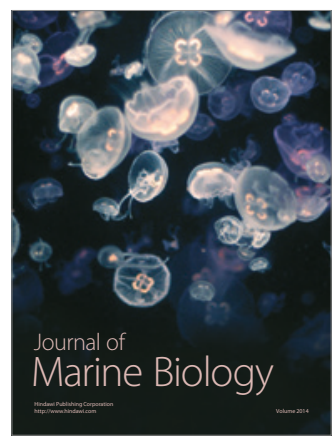

